\title{
Preface to the December 2014 issue
}

\author{
Héctor Cancela \\ Facultad de Ingeniería, Universidad de la República \\ J. Herrera y Reissig 565, Montevideo, Uruguay \\ cancela@fing.edu.uy
}

Since 1998, CLEIej has been the leading electronic, open access journal in Computer Science in Latin America. This pioneering role has enabled the regional community to take advantage from a high-quality, peer-reviewed venue for publishing original research in all areas related to computing.

This issue of CLEIej combines regular papers and papers selected from events. The first four papers in this issue are extended and revised versions of a selected few papers presented at CIbSE 2013, the XVI Ibero-American Conference on Software Engineering, which took place at Montevideo, Uruguay, in April 2013. These papers were selected by invited editors Luca Cernuzzi, Nelly Condori, Diego Vallespir and Martín Solari. The fith paper in this issue is a regular contribution. Finally, we find a selection of works presented at CIESC 2013, the XXI Iberoamerican Congress on Higher Education in Computing, which took place at Naiguatá, Venezuela, in October 2013. This set of six papers were selected by the Program Chairs of the congress, Silvana Roncagliolo and Jonás Montilva, who also provided a short introduction to their selection.

We want to thank the invited editors, the reviewers, and the authors who contributed their work toward this issue of the CLEI electronic journal; we hope the community as a whole will find here a set of interesting research and application ideas, and continue contributing towards our journal, whose main objective is to promote research in Latin America and give visibility to the obtained results. 